\title{
THE AUTOMORPHISM GROUP OF A FREE GROUP IS NOT A CAT(0) GROUP
}

\author{
S. M. GERSTEN
}

(Communicated by Ronald Stern)

\begin{abstract}
If $F$ is a finitely generated free group, then the group $\operatorname{Aut}(F)$, if $\operatorname{rank}(F) \geq 3$, and $\operatorname{Out}(F)$, if $\operatorname{rank}(F) \geq 4$, are not isomorphic to a subgroup of a group which acts properly discontinuously and cocompactly on a 1-connected geodesic metric space satisfying Gromov's condition CAT(0).
\end{abstract}

\section{INTRODUCTION}

In his thesis, Bridson [ $\mathrm{Br} 1]$ established that, for $n \geq 3$, the Culler-Vogtmann space $X\left(F_{n}\right)$, which is a contractible finite-dimensional $\mathrm{CW}$ complex on which $\operatorname{Out}\left(F_{n}\right)$ acts properly, cellularly, and with compact quotient does not admit a piecewise Euclidean metric of nonpositive curvature, which is invariant under the group action; here $F_{n}$ denotes a finitely generated free group of rank $n$. This left open the question whether $\operatorname{Out}\left(F_{n}\right), n \geq 3$, can act properly by isometries on any simply connected geodesic metric space satisfying Gromov's condition $\operatorname{CAT}(0)[\mathrm{Gr}]$ with a compact quotient. The question was originally raised in the attempt to prove that $\operatorname{Out}\left(F_{n}\right)$ is combable (or, more optimistically, automatic), since such an action would give a combing [ECHLPT]. In this note we shall establish the following result.

Theorem. If $F$ is a finitely generated free group, then the group $\operatorname{Aut}(F)$, if $\operatorname{rank}(F) \geq 3$, and $\operatorname{Out}(F)$, if $\operatorname{rank}(F) \geq 4$, are not isomorphic to a subgroup of a group which acts properly, discontinuously, and cocompactly by isometries on a 1-connected geodesic metric space satisfying the condition $\mathrm{CAT}(0)$.

In this connection, we note that $\operatorname{Out}\left(F_{2}\right)=\mathrm{Gl}_{2}(\mathbb{Z})$ acts on a simplicial tree with finite stabilizers [Se]. In addition, $\operatorname{Aut}\left(F_{2}\right)$ is known to be commensurable with the quotient of the braid group $B_{4}$ by its $\mathbb{Z}_{2}$ center and hence is automatic [ECHLPT]. Otherwise it is open whether $\operatorname{Out}\left(F_{n}\right)$ and $\operatorname{Aut}\left(F_{n}\right)$ are either (bi-)automatic or (bi-)combable for $n \geq 3$.

Our result is an application of a geometric result due to Bridson [Br2], which is in turn a generalization of the flat subspace theorem of Gromoll and Wolf

Received by the editors October 30, 1992.

1991 Mathematics Subject Classification. Primary 20F32, 20E05, 53C23, 57M07.

Key words and phrases. Free group, automorphism, CAT(0), geodesic metric space.

This research was partly supported by National Science Foundation grant DMS-9200433. 
[GW] and Lawson and Yau [LY]. Bridson's result states the following (the terms will be explained in the next paragraph).

Theorem (Bridson). If $X$ is a simply connected geodesic metric space satisfying $\mathrm{CAT}(0)$ which is acted on properly discontinuously and isometrically by a group $G$ with compact quotient $G \backslash X$ and if $A<G$ is a free Abelian subgroup of rank $r$, then there is a flat subspace $Y$ isometric to $\mathbb{R}^{r}$ which is isometrically and totally geodesically embedded in $X$ and such that $Y$ is stabilized by $A$.

We recall here that a metric space $X$ is called geodesic if for any two points $x, y$ of $X$ there is an isometric embedding of an interval $f:[a, b] \rightarrow X$ with $f(a)=x, f(b)=y$, and $d(x, y)=b-a$. There are various equivalent ways of formulating the condition $\operatorname{CAT}(0)$ for a geodesic metric space [ $\mathrm{Br} 1, \mathrm{GH}]$. For definiteness, we adopt the following definition. Let $\Delta=[x, y, z]$ be a geodesic triangle in $X$, so the sides $[x, y],[y, z]$, and $[z, x]$ are geodesic segments. Let $\Delta^{\prime}=\left[x^{\prime}, y^{\prime}, z^{\prime}\right]$ be a comparison triangle in $\mathbb{R}^{2}$ so that corresponding sides of $\Delta$ and $\Delta^{\prime}$ are of equal length. We require that if $p$ is any point on $[y, z]$ and $p^{\prime}$ is the corresponding point on $\left[y^{\prime}, z^{\prime}\right]$ (so $p^{\prime}$ divides the segment $\left[y^{\prime}, z^{\prime}\right]$ in the same ratio of lengths as $p$ divides $[y, z])$, then $d(x, p) \leq d^{\prime}\left(x^{\prime}, p^{\prime}\right)$, where $d^{\prime}$ denotes the Euclidean metric of $\mathbb{R}^{2}$.

To say that $Y$ is totally geodesically embedded in $X$ in the statement of the theorem means that the (unique) geodesic in $X$ connecting any two given points of $Y$ lies entirely in $Y$.

As an example, if $M$ is a closed Riemannian manifold with all sectional curvatures nonpositive, then the universal cover $\widetilde{M}$ is a geodesic metric space satisfying $\operatorname{CAT}(0)$. For examples of $\operatorname{CAT}(0)$ spaces which are not manifolds, see $[\mathrm{Br} 1]$.

\section{TRANSLATION LENGTHS}

We assume in this section that $X$ is a 1-connected geodesic metric space which is acted on properly discontinuously by a discrete group $G$ of isometries so that the quotient $G \backslash X$ is compact. In addition we assume that $X$ satisfies $\operatorname{CAT}(0)$.

If $g \in G$ is of infinite order, then by Bridson's theorem $g$ stabilizes a flat $\mathbb{R}^{1}$ isometrically embedded in $X$. Thus $g$ acts on the flat by translation by a positive real number $\tau_{\text {geo }}(g)$, which is defined to be infimum of the displacement function $x \mapsto d(x, g x), x \in X$. Thus the number $\tau_{\text {geo }}(g)$ is independent of the flat $\mathbb{R}^{1}$ stabilized by $g$. This can be seen geometrically by using the fact, proved in the course of establishing Bridson's theorem, that two such flats stabilized by $g$ cobound a flat strip bounded by parallel straight lines and stabilized by $g$. From the fact that $g$ acts isometrically, it follows that $g$ translates one boundary $\mathbb{R}^{1}$ the same amount as it translates the other.

The function $\tau_{\text {geo }}$ satisfies the following properties, which are established easily from the definition:

(1) $\tau_{\text {geo }}(g)=\tau_{\text {geo }}\left(h g h^{-1}\right)$ for all $g, h \in G$.

(2) $\tau_{\text {geo }}\left(g^{n}\right)=|n| \tau_{\text {geo }}(g)$ for $n \in \mathbb{Z}$.

If $A<G$ is a free abelian subgroup of $\operatorname{rank} r$, let $Y \subset X$ be a flat $\mathbb{R}^{r}$ stabilized by $A$. Since $Y$ is isometrically and totally geodesically embedded in $X$, it follows that the translation numbers of elements of $A$ calculated in 
$\mathbb{R}^{r}$ and $Y$ are the same. If we choose a point $y \in Y \cong \mathbb{R}^{r}$ as the origin, then $a \in A$ can be identified with its displacement vector $v_{a}$ at the origin, since the group $A$, considered as a group of isometries $\mathbb{R}^{r}$, consists only of translations. One has the equality $\tau_{\text {geo }}(a)=\left\|v_{a}\right\|$, where $\|v\|$ denotes the Euclidean norm of the vector $v \in \mathbb{R}^{r}$.

Let $F=F(a, b, c)$ be the free group freely generated by $a, b$, and $c$, and let $\phi: F \rightarrow F$ be the automorphism given by $a \mapsto a, b \mapsto b a, c \mapsto c a^{2}$. Thus the split extension $H=F \rtimes_{\phi} \mathbb{Z}$ has presentation

$$
\mathscr{P}=\left\langle a, b, c, t \mid t a t^{-1}=a, t b t^{-1}=b a, t c t^{-1}=c a^{2}\right\rangle .
$$

Proposition 2.1. The group $H$ above is not isomorphic to a subgroup of any group $G$ of isometries acting properly discontinuously on a 1-connected geodesic metric space $X$ with compact quotient $G \backslash X$ where $X$ satisfies $\mathrm{CAT}(0)$.

Proof. Observe that the second and third relations of $\mathscr{P}$ can be rewritten as $b^{-1} t b=a t$ and $c^{-1} t c=a^{2} t$. By the first relation of $\mathscr{P}, A:=\langle a, t\rangle$ is free abelian of rank 2 .

If $H<G$, where $G$ acts properly discontinuously on the 1-connected geodesic metric space $X, G \backslash X$ is compact, and $X$ satisfies CAT(0), then the function $\tau_{\text {geo }}$ associated to $G$ and $X$ satisfies $\tau_{\text {geo }}(t)=\tau_{\text {geo }}(a t)=\tau_{\text {geo }}\left(a^{2} t\right)$, where we have used property (1) of translation numbers. By Bridson's theorem, $A$ stabilizes a flat $\mathbb{R}^{2}$. If we pick a base point in this flat, then $t, a$ can be identified with translations by independent vectors $v_{t}, v_{a}$ in this flat. Hence we have $\left\|v_{t}\right\|=\left\|v_{t}+v_{a}\right\|=\left\|v_{t}+2 v_{a}\right\|$. But these equalities cannot be satisfied for two independent vectors $v_{t}, v_{a} \in \mathbb{R}^{2}$, since they say that a line intersects a circle in 3 points. This contradiction shows that $H$ cannot be isomorphic to a subgroup of $G$.

Question. We would like to know whether or not the group $H$ is (bi-)combable (resp. (bi-)automatic). Bestvina informed us that it follows from his joint work with Feighn $[\mathrm{BF}]$ that $H$ satisfies the quadratic isoperimetric inequality, so this can be taken as positive evidence.

Theorem 2.2. Each of the following groups cannot be isomorphic to a subgroup of a group $G$ which acts properly discontinuously by isometries with compact quotient on a 1-connected geodesic metric space satisfying $\mathrm{CAT}(0)$ :

(1) $\operatorname{Aut}\left(F_{n}\right)$ if $n \geq 3$, and

(2) $\operatorname{Out}\left(F_{n}\right)$ if $n \geq 4$.

Proof. We let $H=F(a, b, c) \rtimes_{\phi} \mathbb{Z}$, where $\phi: a \mapsto a, b \mapsto b a, c \mapsto c a^{2}$. We embed $H$ in $\operatorname{Aut}(F(a, b, c))$ as follows. Let $F=F(a, b, c)$, and let $\imath: F \rightarrow \operatorname{Aut}(F)$ send $f \in F$ to the inner automorphism $l_{f}$, so $l_{f}(x)=f x f^{-1}$, for $x \in F$. With $\phi$ as above, one checks that $\phi l_{f} \phi^{-1}=l_{\phi(f)}$, which produces a homomorphism $H \rightarrow \operatorname{Aut}(F)$. This homomorphism is easily seen to be injective. Hence $H$ embeds in $\operatorname{Aut}\left(F_{3}\right)$. Since $\operatorname{Aut}\left(F_{3}\right)$ embeds in $\operatorname{Aut}\left(F_{n}\right)$ for all $n \geq 3$, we have embeddings of $H$ in $\operatorname{Aut}\left(F_{n}\right)$ for all $n \geq 3$.

Next one observes that $\operatorname{Aut}\left(F_{n}\right)$ embeds in $\operatorname{Out}\left(F_{n+1}\right)$, by stabilizing the last element of a free basis. Thus $H$ embeds in $\operatorname{Out}\left(F_{n}\right)$ for all $n \geq 4$.

The result follows from these observations by applying Proposition 2.1 .

Remark. It is interesting to compare our result with that of [FP], which gives the same values of $n$ for which $\operatorname{Aut}\left(F_{n}\right)$ and $\operatorname{Out}\left(F_{n}\right)$ are not linear. 


\section{REFERENCES}

[Br1] M. R. Bridson, Geodesics and curvature in metric simplicial complexes, Group Theory from a Geometrical Viewpoint (E. Ghys, A. Haefliger, and A. Verjovsky, eds.), World Scientific, 1990, pp. 373-463.

[Br2] M. R. Bridson, $A$ generalized flat torus theorem with applications (in preparation).

[BF] M. Bestvina and M. Feighn, A combination theorem for negatively curved groups, Differential Geom. 35 (1992), 85-101.

[ECHLPT] D. B. A. Epstein, J. W. Cannon, D. F. Holt, S. V. F. Levy, M. S. Paterson, and W. P. Thurston, Word processing in groups, Bartlett and Jones, Boston, MA, 1992.

[FP] E. Formanek and C. Procesi, The automorphism group of a free group is not linear, J. Algebra 149 (1992), 494-499.

[Gr] M. Gromov, Hyperbolic groups, Essays in Group Theory (S. M. Gersten, ed.), Math. Sci. Res. Inst. Publ., vol. 8, Springer-Verlag, New York and Berlin, 1987.

[GH] E. Ghys and P. de la Harpe, Notes sur les groupes hyperboliques de Mikhael Gromov, Birkhäuser, Boston, MA, 1990.

[GW] D. Gromoll and J. A. Wolf, Some relations between the metric structure and the algebraic structure of the fundamental group in manifolds of nonpositive curvature, Bull. Amer. Math. Soc. 77 (1971), 545-552.

[LY] H. B. Lawson and S. T. Yau, Compact manifolds of nonpositive curvature, J. Differential Geom. 7 (1972), 211-228.

[Se] J.-P. Serre, Trees, Springer-Verlag, New York, 1980.

Department of Mathematics, University of Utah, Salt Lake City, Utah 84112

E-mail address: gersten@math.utah.edu 